\title{
Perception of Waiting Time at Signalized Intersections
}

\author{
Xinkai Wu, David M. Levinson, and Henry X. Liu
}

Perceived waiting time at signalized intersections differs from the actual waiting time and varies with signal design. The onerousness of delay depends on the conditions under which it is experienced. Using weighted travel time may contribute to optimal signal control if it can improve on the assumption that all time is weighted equally by users. This research explores the perception of waiting time at signalized intersections on the basis of the results of an online virtual experience stated preference survey. This survey directly collected the perceived waiting times and the user ratings of the signal designs of each intersection on an arterial that included three intersections. Statistically analyzing the survey data suggests that the perception of waiting time is a function of the actual time, and a quadratic model describes the relationship. The survey also indicates that there exists a trade-off between total waiting time and individual waiting time at each intersection. Drivers prefer to split the total waiting time across multiple intersections at the price of a longer total wait if the difference in the total waiting time of two signal designs is within $30 \mathrm{~s}$. Survey data show that the perceived waiting time, instead of the actual waiting time, better explains how users rate the individual signal designs for intersections and arterials, including multiple intersections.

Traditionally, traffic engineers operate signalized intersections to satisfy engineering efficiency objectives. Generally, they minimize the total travel time (delay), subject to maximum wait time constraints, a strategy that benefits major streets to the detriment of minor approaches. This policy may leave a vehicle on a minor street stopped and waiting for more than 2 to $3 \mathrm{~min}$. As a result of such excessive waiting time, drivers may come to believe that a signal is malfunctioning, leading them to violate signal control. For this reason, many intersections have a fixed cycle length, but that may not be optimal. Many optimization methods try to improve users' satisfaction but all of them assume that waiting time is equivalent independent of conditions.

Intuitively, the value of travel time is not a constant. It varies for each trip and, more generally, differs by trip purpose, trip distance, mode, and departure time as well as by individual and may be associated with characteristics like income, gender, and personality. Most important, signal control affects the perceived travel time. Specifically, people might be unwilling to wait on any one street for a very long time, even if total waiting time has decreased. In other words, the perception of waiting time may change with signal design.

Department of Civil Engineering, University of Minnesota, 500 Pillsbury Drive Southeast, Minneapolis, MN 55455. Corresponding author: X. Wu, wuxxx273@ umn.edu.

Transportation Research Record: Journal of the Transportation Research Board, No. 2135, Transportation Research Board of the National Academies, Washington, D.C., 2009, pp. 52-59.

DOI: 10.3141/2135-07
To satisfy users, signal design would be based on drivers' perceived instead of observed travel time. However, it is an extremely difficult problem on which no current literature can be found. Researchers have begun to pay attention to the perception of travel time on ramps and freeways (1), but no similar studies have been conducted for signalized intersections.

This paper reports on a study to explore the perceived waiting time at signalized intersections. Several questions are answered: what is the relationship between the perceived waiting time and the actual waiting time; what is the trade-off between the overall waiting time and the waiting time at each intersection; and what better explains user preference, perceived or observed waiting time? The answers given in this study are based on statistically analyzing the results of an online, virtual experience stated preference (SP) survey of the perception of waiting for signal control.

The paper is organized as follows. The background of perception of travel time is presented in the next section, followed by a survey description. The results are analyzed in the fourth section, and the final section describes conclusions and future research.

\section{BACKGROUND}

The variable perception of waiting time has been observed in psychology. Loehlin mentioned that time in idleness, particularly waiting in idleness, is perceived as longer than the real time (2). The psychological reason is that, after waiting for a certain amount of time, "anxiety and stress start to build up in an individual, due both to the sense of waste and the uncertainty involved in a waiting situation" (3, p. 82). The uncertainty is "anxiety provoking and as such can be considered as an agent for psychological stress" (4, p. 443). So, the intensity and duration of exposure to this situation will be "responsible for the psychological stress and its resulting effects of anxiety and uneasiness on the individual" (3, p. 83). Psychological stress, or cost, will accumulate during the waiting process. Theoretically, Osuna found that "stress intensity increases during the waiting process, and consequently that the psychological cost of waiting is a marginal increasing function of waiting time" using general probability theory $(3, \mathrm{p} .83)$.

Economically, travel time has value. Becker's time allocation theory postulates that individual satisfaction came from the "final commodities" that use market goods and time as inputs instead of goods consumed directly (5). This theory introduced a time constraint in addition to the usual income constraint. According to Becker, time could be converted into money by assigning less time to consumption and more time to work. Later, Johnson (6), Oort (7), and Evans (8) pointed out that time (at the margin) has a single value for all activities. DeSerpa (9) first proposed a utility function to search a set of 
TABLE 1 Scenarios and Statistical Results of Rating for Four Scenarios

\begin{tabular}{|c|c|c|c|c|c|c|c|c|c|c|}
\hline & \multicolumn{4}{|c|}{ Waiting Time (s) } & \multicolumn{2}{|c|}{ First Survey } & \multicolumn{2}{|c|}{ Second Survey } & \multicolumn{2}{|c|}{ Combined } \\
\hline & $T_{A 1}$ & $T_{A 2}$ & $T_{A 3}$ & Total $T_{A}$ & Mean & Std. Dev. & Mean & Std. Dev. & Mean & Std. Dev. \\
\hline Scenario 1 & 120 & 0 & 0 & 120 & 2.57 & 1.78 & 3.93 & 2.07 & 3.39 & 2.04 \\
\hline Scenario 2 & 90 & 20 & 20 & 130 & 3.83 & 2.04 & 4.86 & 1.52 & 4.39 & 1.75 \\
\hline Scenario 3 & 60 & 40 & 40 & 140 & 4.04 & 1.65 & 4.23 & 2.14 & 4.13 & 1.89 \\
\hline Scenario 4 & 30 & 60 & 60 & 150 & 3.52 & 2.29 & 3.89 & 1.68 & 3.73 & 1.96 \\
\hline
\end{tabular}

NotE: $T_{A}=$ actual waiting time; std. dev. = standard deviation

minimum time requirements for each activity explicitly. In this model, the level of utility depends on the consumption of all goods and on the time assigned to all activities (10). Because the reduction in travel time has a direct effect, an exogenous reduction in travel time changes the utility (11).

One interesting application is to evaluate the value of time in transportation. Bruzelius demonstrated that people value a saving in waiting time much higher (about three times) than in-vehicle travel time (12). Recently, Levinson et al. explored the waiting tolerance between ramp stop delay and freeway congestion delay $(1,13)$. In that study, the SP data were collected by asking drivers to rate and rank four choice conditions with different ramp meter wait times. Two survey methods were used: a traditional computer-administered SP questionnaire and a driving simulator to collect virtual experience SP survey data. Use of a rank-ordered logit model, however, gave two different results. The traditional computer-administered SP data suggested that ramp delay is 1.6 to 1.7 times more onerous than delay on freeways, whereas the virtual experience SP data suggested that freeway delay is more onerous than ramp delay (1) and applied a binary logit model to analyze the results statistically.

Several reasons are provided in this research to explain why the results of the two surveys are significantly different. Using the same database Zhang et al. estimated the subjective value of travel time on freeways and ramp meters by using utility function based on the computer-administered SP survey data (14). This research indicated that drivers perceive ramp wait as more onerous than freeway travel. A possible approach, which aims to minimize total perceived travel time instead of total system delay, is suggested by the authors to deal with the problem of variation in the subjective value of travel time, which can be used in ramp control (15).

This situation suggests there is valuable research to be done to evaluate the variable perception of waiting time on major and minor streets of signalized intersections. This paper aims to address the issue with an online survey using virtual experience SP methodology.

\section{ONLINE SURVEY}

To obtain information about perceived waiting time, surveys are required, as this value cannot be directly measured. Finding a realworld circumstance to conduct such an experiment is difficult because of variable conditions and the difficulty in having to stop drivers immediately to garner perceptions; putting a surveyor in the car with the driver would be expensive and less realistic. A virtual experience SP, putting drivers in the driver seat of a virtual car, has advantages in this regard, as the situation can be highly controlled. Some virtual environments are more expensive than others; to keep costs low, the driving environment here was a simulated movie from the driver's perspective presented on a computer screen. Although this environment is not ideal, much more expensive driving simulators also pose difficulties (1).

In this survey, four scenarios were designed (Table 1), representing the same physical environmental: an arterial including three intersections and two links. The traffic conditions were assumed to be free flow between intersections; drivers will accelerate to free-flow conditions from a stop and decelerate before a stop. The wait times at red lights are as shown in Table 1 . The route of the subject's virtual vehicle is predesigned. The vehicle starts from Intersection I from a minor street, turns right (no turn on red is designed in the study), and then continues on that road passing two intersections on the major streets (Figure 1). Vehicles may stop before Intersection I, II, or III depending on the scenario. The travel situations are vividly presented by several three-dimensional traffic simulation videos generated by AIMSUN, a commercial traffic microsimulator (Figure 2).

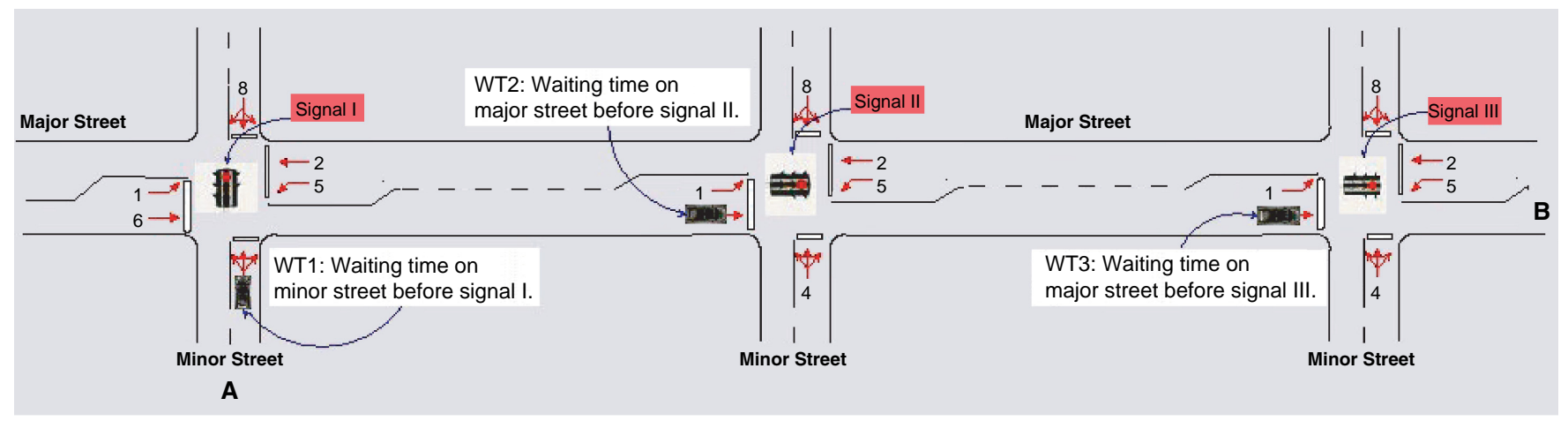

FIGURE 1 Layout of signalized intersections (total waiting time $=$ WT1 + WT2 + WT3). 


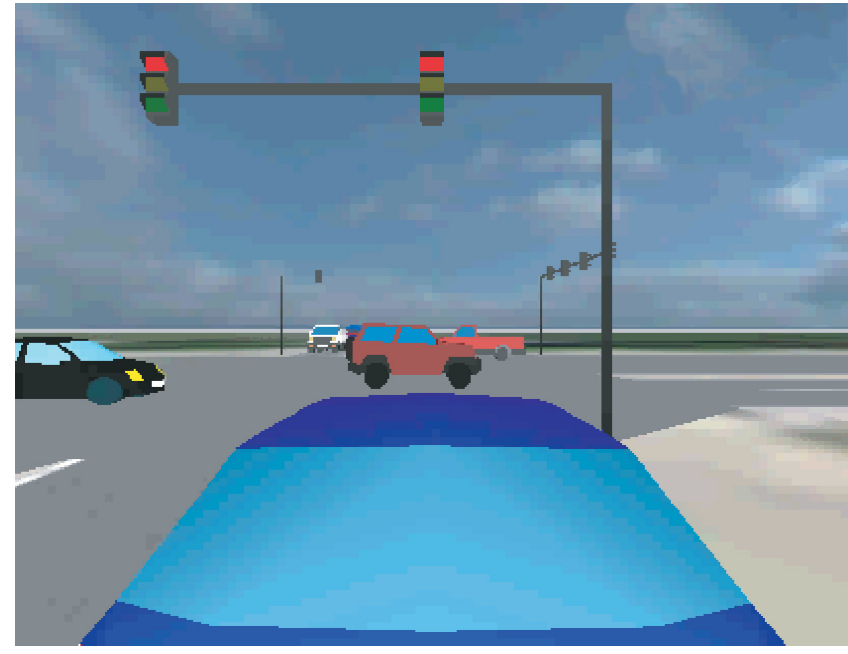

(a)

FIGURE 2 Three-dimensional traffic microsimulation.

The four scenarios were specifically designed to explore the trade-off between different signal settings. As indicated on the left side of Table 1, the total waiting time of these scenarios varies from 120 to $150 \mathrm{~s}$. Scenario 1 has the shortest total waiting time but requires drivers to stop as long as $120 \mathrm{~s}$ at the first intersection. The other scenarios have longer total waiting times but a relatively shorter waiting time at each intersection. The trade-off is made for users to wait $2 \mathrm{~min}$ at one intersection to reduce total time by 10 or $20 \mathrm{~s}$ or sacrifice 10 or $20 \mathrm{~s}$ to obtain a relatively shorter waiting time at later intersections.

In the first survey, 76 University of Minnesota undergraduate students in CE3201: Introduction to Transportation Engineering were invited to take part in the survey, and 66 students participated. CE3201 is generally the first or second engineering course students take; most have not formed professional opinions on perceived travel time (which is not covered in the class) or signal design, which had not been taught yet, and it is believed that these students perceive the world much as other young drivers do. In the second survey, conducted in fall 2008, this survey was expanded to a more general population. Of 5,000 individuals invited to participate, 329 subjects responded and 88 completed the survey. Among these records, only 69 are useful, as some data were not successfully recorded. The first and second surveys as well as combined data are analyzed here.

Demographic information including gender, age, education, driving experience (years of driving), and driving attitude (enjoy or not) were collected in both surveys. These data are presented in Table 2. Two groups have different social demographic statistics. The group in the first survey is much younger (average age 21 years) than the second group (average age 42 years), and the proportion of females is lower in the first than in the second group. Almost all subjects in the second survey have more than 5 years of driving experience, compared with one-third in the first survey. Most subjects in both surveys enjoy driving.

Participants were asked to rate the overall signal system for the entire route. The rating varies from 0 (worst) to 9 (best). In addition, to learn the perception of waiting time at each intersection, participants were required to estimate the waiting time for each intersection after they watched the video and evaluated this specific intersection signal

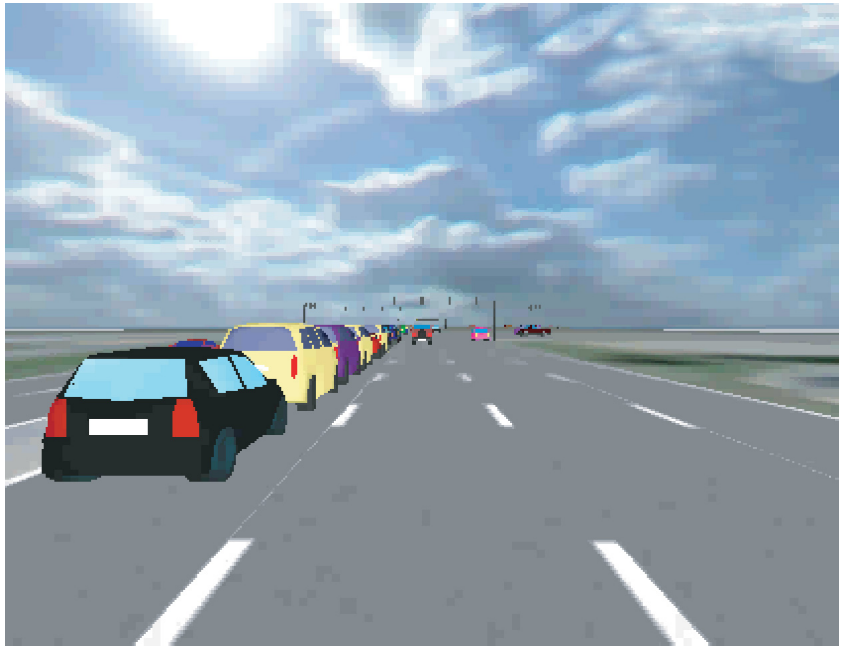

(b) setting based on their satisfaction (Figure 3). As the waiting time is being evaluated, participants may be required to watch the video with largely unchanged scenes for a long time (e.g., 2 min for Scenario 1) (though there is traffic that users can detect passing across the intersection ahead of them); each scenario has the same background. To avoid order and fatigue effects and improve the reliability of the survey data, only two scenarios, which are randomly selected, are presented in the survey to each subject.

\section{DATA ANALYSIS AND HYPOTHESIS TESTING}

\section{Perceived Waiting Time}

The survey directly provides the perceived $\left(T_{P}\right)$ and actual $\left(T_{A}\right)$ waiting times. The comparisons based on the combined data are presented in Figure 4. $T_{P}$ varies widely around the actual $T_{A}$. Consistently, for both the first and second surveys, the average $T_{P}$ is lower than the true value in most situations, but the standard deviation increases with the true value of $T_{A}$ (Figure 5). Figure 5 shows that if $T_{A}$ is small $(<100 \mathrm{~s})$, drivers tend to underestimate it; however, this estimate is close to the actual value when $T_{A}$ is $120 \mathrm{~s}$. These data indi-

TABLE 2 Descriptive Statistics of Survey Subjects

\begin{tabular}{|c|c|c|c|}
\hline Variable & $\begin{array}{l}\text { First } \\
\text { Survey } \\
\text { Count }^{a}\end{array}$ & $\begin{array}{l}\text { Second } \\
\text { Survey } \\
\text { Count }^{b}\end{array}$ & $\begin{array}{l}\text { Combined } \\
\text { Count }^{c}\end{array}$ \\
\hline Female/male & $12 / 54$ & $46 / 23$ & $58 / 77$ \\
\hline $\begin{array}{l}\text { Education: 4-year college } \\
\text { and above/other }\end{array}$ & $20 / 46$ & $38 / 31$ & $58 / 77$ \\
\hline $\begin{array}{l}\text { Driving experience: more than } \\
5 \text { years/less than or equal } \\
\text { to } 5 \text { years }\end{array}$ & $24 / 42$ & $68 / 1$ & $92 / 43$ \\
\hline Enjoy driving/not & $61 / 5$ & $63 / 6$ & $124 / 11$ \\
\hline
\end{tabular}

${ }^{a}$ Mean age $=21$ years; standard deviation $=3$ years.

${ }^{b}$ Mean age $=42$ years; standard deviation $=12$ years.

${ }^{c}$ Mean age $=32$ years; standard deviation $=14$ years 


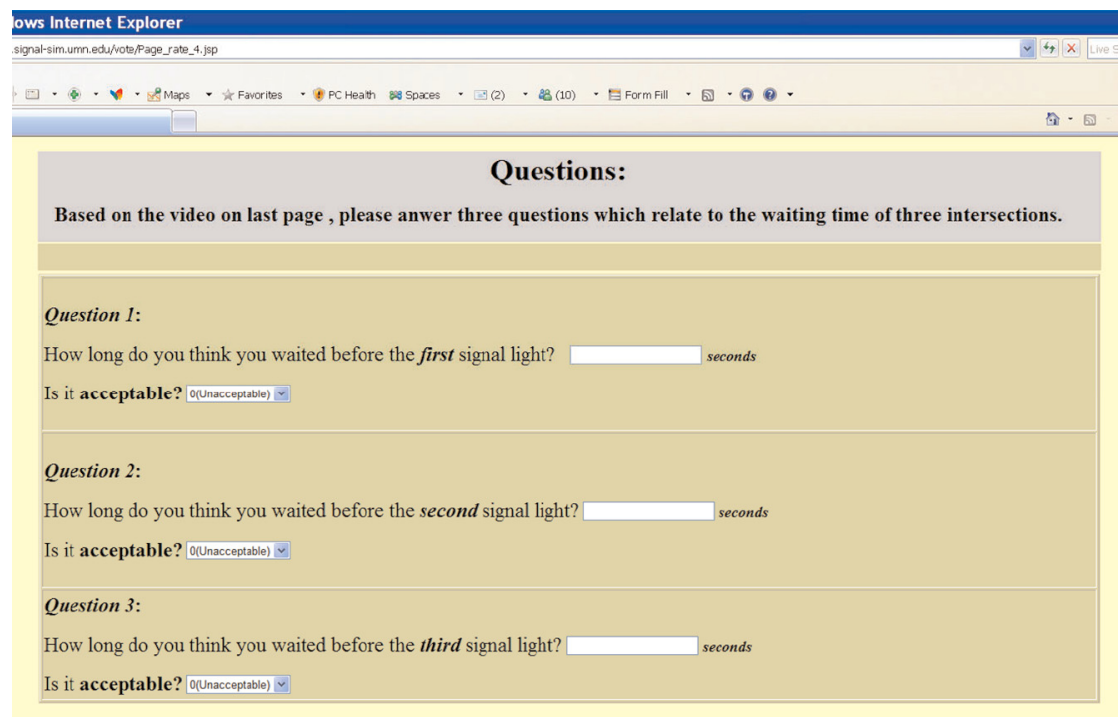

Next

FIGURE 3 Perceived waiting time questions.

cate that participants actually overestimate the actual value when $T_{A}$ is $120 \mathrm{~s}$. By extrapolating the curves in Figure 5, it is anticipated that the average perceived time would be larger than the true time if this value were larger. Actually, the increase in variance also indicates this point. As shown in Figure 4, when the waiting time is $120 \mathrm{~s}$, a large share of participants perceived $T_{A}$ to be much longer than the true value.

The common argument about perceived travel time is that the perceived value is a nonlinear function of actual travel. Two simple models, which consider the sociodemographic variables, are proposed to test this assumption with the combined data.

Model 1:

$T_{P}=\beta_{01}+\beta_{\mathrm{S} 1} \mathbf{S}+\beta_{11} T_{A}+\beta_{21} D$
Model 2:

$$
T_{P}=\beta_{02}+\beta_{\mathrm{S} 2} \mathbf{S}+\beta_{12} T_{A}+\beta_{22} T_{A}^{2}+\beta_{32} D
$$

where

$\beta=$ coefficient;

$\mathbf{S}=$ vector of sociodemographic variables composed of age, gender, level of education, driving experience, and driving attitude (enjoy or not); and

$D=$ dummy variable with 0 representing the first survey data set and 1 representing the second.

$D= \begin{cases}0 & \text { first survey data set } \\ 1 & \text { second survey data set }\end{cases}$

(Sec)

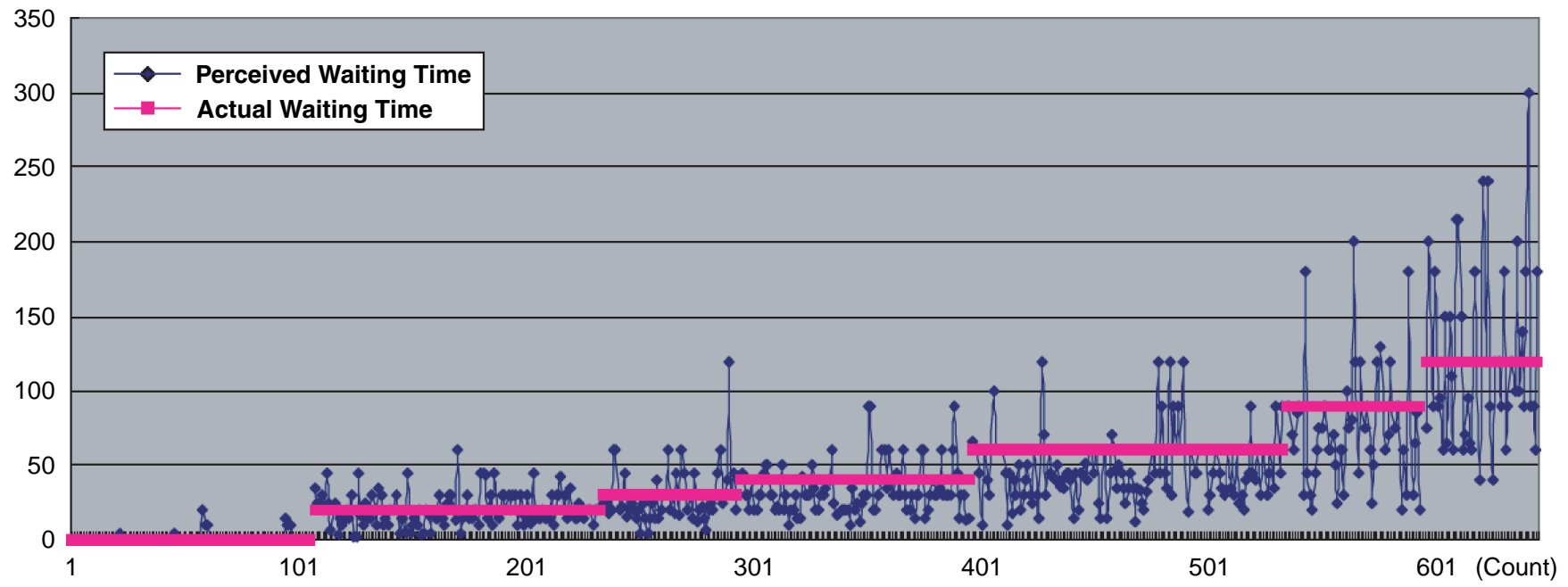

FIGURE 4 Perceived waiting time and actual waiting time. 


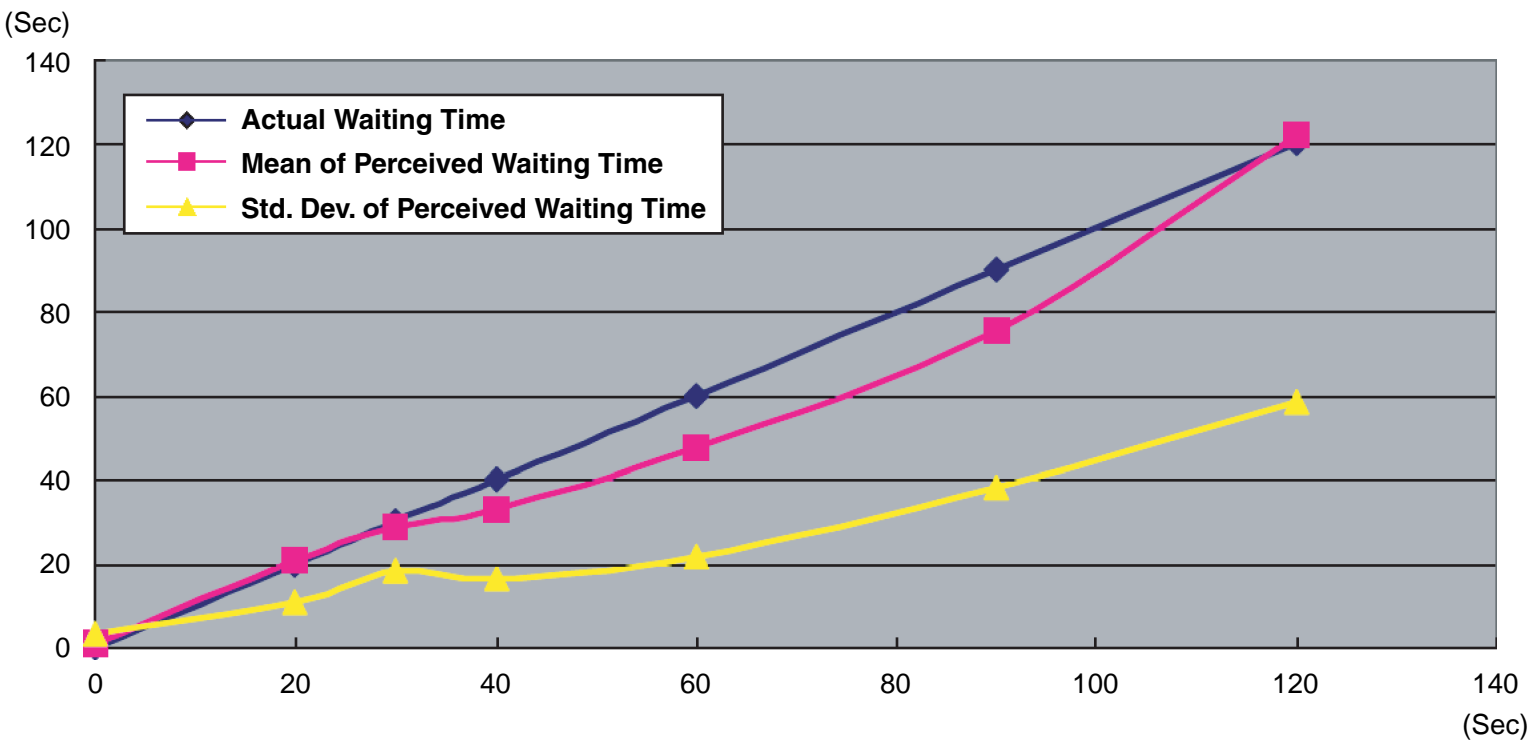

FIGURE 5 Mean and standard deviation (std. dev.) of perceived waiting time.

The model summaries are presented in Table 3. Although all models are significant at the $95 \%$ confidence interval, the quadratic model (Model 2) has a slightly better fit than the linear model (Model 1) as indicated by comparing adjusted $R^{2}$ values. In addition, the sociodemographic variables are not significant in the two models based on $t$-values. But the dummy variable indicates that the two data sets are significantly different, and the older population perceives a longer travel time.

\section{Trade-Off}

It is hypothesized that there is a trade-off between satisfaction at a single intersection and satisfaction along an arterial. In other words, people may prefer a longer overall waiting time to a shorter one with an extremely long wait at one intersection. As no related literature could be found to deal this problem, the most important objective of

TABLE 3 Perceived Waiting Time Model Summary

\begin{tabular}{lccccc}
\hline & \multicolumn{3}{l}{ Model 1 } & & \multicolumn{2}{l}{ Model 2 } \\
\cline { 2 - 3 } \cline { 6 - 6 } Variable & Coefficient & $t$-Statistic & & Coefficient & $t$-Statistic \\
\hline Constant & -9.20 & -1.75 & & -3.12 & -0.527 \\
Gender & 2.02 & 0.846 & & 1.95 & 0.824 \\
Age & 0.015 & 0.126 & & -0.016 & 0.135 \\
Education & -2.47 & -1.03 & & -2.2 & -0.927 \\
Driving & -1.77 & -0.533 & & -1.19 & -0.36 \\
Enjoy & 4.2 & 1.04 & & 3.72 & 0.929 \\
$T_{A}$ & $\mathbf{0 . 9 2 2}$ & $\mathbf{3 1 . 6}$ & & $\mathbf{0 . 6 1 6}$ & $\mathbf{6 . 9 2}$ \\
$T_{A}^{2}$ & & & $\mathbf{0 . 0 0 3}$ & $\mathbf{3 . 6 5}$ \\
Indicator for & $\mathbf{9 . 3 9}$ & $\mathbf{2 . 4 9}$ & $\mathbf{9 . 2 9}$ & $\mathbf{2 . 4 9}$ \\
\multicolumn{1}{c}{ survey $(D)$} & & & & &
\end{tabular}

NOTE: Bold indicates statistical significance at $95 \%$ confidence interval; significance (two-tailed) for Model 1 is .000 and for Model 2 is .000; adjusted $R^{2}$ for Model 1 is .620 and for Model 2 is .670. this survey is to explore whether this trade-off exists. As the survey also requires participants to evaluate each signal strategy described in Table 1 , the ratings were used to test whether Scenario 1 is significantly worse than the others; it has the shortest total wait but an extremely long wait at the first intersection, whereas the other three have longer total waiting time but the time was assigned to three intersections.

The mean differences and standard deviations for three data sets are presented on the right side of Table 1. Overall, participants in the second survey rated all scenarios slightly higher than the subjects in the first survey. By simply comparing the mean values, it is clear that Scenario 1 is worse than Scenarios 2 and 3 as it has the lowest average rating despite also having the lowest overall travel time in the three data sets. Although the first survey indicates that Scenario 2 is the best, the second survey and the combined data indicate that Scenario 3 has the highest mean rating. More rigorous $t$-statistic tests were applied to test the significance of the following three hypotheses:

Hypotheses 1: $\begin{array}{r}H_{0}: \text { Scenario } 1 \text { has no significant difference } \\ \text { with Scenario } 2 \\ H_{1}: \text { otherwise }\end{array}$
Hypotheses $2:\left\{\begin{array}{r}H_{0}: \text { Scenario } 1 \text { has no significant difference } \\ \text { with Scenario } 3 \\ H_{1}: \text { otherwise }\end{array}\right.$
Hypotheses $3:\left\{\begin{array}{c}H_{0}: \text { Scenario } 1 \text { has no significant difference } \\ \text { with Scenario } 4 \\ H_{1}: \text { otherwise }\end{array}\right.$

Results presented in Table 4 indicate that Hypothesis 1 can be significantly rejected for all data sets as their $t$-values are $>2$ - which means that, instead of waiting for an extremely long time (120 s) at one intersection, drivers prefer to wait at several intersections for a shorter time even if the overall time is longer. The results indicate that, although the actual total wait is shorter for Scenario 1 than for 
TABLE $4 \quad t$-Test

\begin{tabular}{lccccc}
\hline & $t$ & df & $\begin{array}{l}\text { Sig. } \\
\text { (two-tailed) }\end{array}$ & $\begin{array}{l}\text { Mean } \\
\text { Difference }\end{array}$ & $\begin{array}{l}\text { Standard Error } \\
\text { Difference }\end{array}$ \\
\hline First Survey Data & & & & & \\
\hline Scenario 1 versus Scenario 2 & $-\mathbf{2 . 2 0}$ & 43 & .033 & -1.26 & .574 \\
Scenario 1 versus Scenario 3 & $-\mathbf{2 . 9 5}$ & 46 & .005 & -1.47 & .497 \\
Scenario 1 versus Scenario 4 & -1.56 & 46 & .125 & -.947 & .607 \\
\hline Second Survey Data & & & & & .452 \\
\hline Scenario 1 versus Scenario 2 & $-\mathbf{2 . 1 0}$ & 61 & .044 & -.929 & .573 \\
Scenario 1 versus Scenario 3 & -0.527 & 52 & .600 & -.302 & .472 \\
Scenario 1 versus Scenario 4 & -0.091 & 61 & .928 & -.043 & .354 \\
\hline Combined Data & & & & & .386 \\
\hline Scenario 1 versus Scenario 2 & $-\mathbf{2 . 8 2}$ & 113 & .006 & -.998 & .377 \\
Scenario 1 versus Scenario 3 & -1.92 & 102 & .058 & -.740 & -.334 \\
Scenario 1 versus Scenario 4 & -0.760 & 111 & .379 & & \\
\hline
\end{tabular}

NOTE: Bold indicates statistical significance at $95 \%$ confidence interval; $\mathrm{df}=$ degrees of freedom, sig. $=$ significance.

Scenario 2, the perceived time of Scenario 1 is significantly longer than for the other two.

The first survey data also indicate that Hypothesis 2 can be rejected, but this conclusion is not supported by the second survey and the combined data. For both data sets (and the combined data), Hypothesis 3 cannot be significantly rejected, which means that Scenario 1 is not significantly worse than Scenario 4. This finding helps pinpoint the trade-off threshold; the difference in the total waiting time for Scenario 1 and Scenario 4 is as high as 30 s, 25\% of the total waiting time of Scenario 1 . This huge gap has been considered when people evaluate these two scenarios. This result also indicates the rough bound of the trade-off. If the difference between two signal designs in terms of total waiting time is less than $30 \mathrm{~s}$, $95 \%$ of drivers prefer to split the total waiting time among different intersections, although the overall waiting time is longer; however, if the difference is greater than $30 \mathrm{~s}$, different drivers may make different decisions for other reasons. This finding is very important for signal design. Much current practice aims to minimize the total travel time subject to constraints. This optimum may not be a "real" optimum because this kind of design intends to sacrifice travel time on minor streets to achieve a minimum total waiting time for the whole system. The extremely long waiting time on the minor street significantly decreases user satisfaction, so from the perspective of overall $T_{P}$, the signal is not optimal.

There is an interesting phenomenon worth noting. Among 88 participants in the second survey, 39 left comments and 11 mentioned that they preferred fewer or no stops (about 28\%). Although these subjects may "feel" more comfortable when they experience a signal design that spreads total waiting time among different intersections as indicated by higher ratings of Scenario 2, these comments suggest that the number of stops should be seriously considered in signal timing design. This finding may also explain that why Hypothesis 2 cannot be significantly rejected in the second survey, as a relatively long waiting time (more than $30 \mathrm{~s}$ ) at each intersection for Scenario 3 aggravates the uncomfortable feeling of "stopping at each intersection" and leads to low ratings. Unfortunately, current survey design is not able to identify the effect of stops. This topic is left for future study.

\section{Model of Rating}

The preceding testing results imply that $T_{P}$, rather than $T_{A}$, more accurately reflects user satisfaction. User ratings are used here to fit ordinary least-squares regression models to compare. The participants in this survey were asked not only to evaluate the whole signal design for the arterial but also to evaluate the signal setting of each intersection to find which intersection is the crucial one that affects user satisfaction. These ratings (for the arterial and for each intersection) are used to build two sets of models. The first one is at the individual intersection level, which uses the ratings of each intersection to build a model to describe the relationship between user satisfaction (i.e., the ratings) and $T_{P}$ or $T_{A}$; the second is at the arterial level, which uses the ratings for the arterial to build a model to describe the relationship between satisfaction and $T_{P}$ and $T_{A}$. The statistical testing results for the combined data are presented in the following sections.

\section{Individual Intersection Level}

Two ordinary least-squares regression models, one based on $T_{A}$ and the other based on $T_{P}$, are fit here. These ratings of individual intersection signal design are used. The sociodemographic and attitude variables are considered in the models. To distinguish the impact of different intersections, two dummy variables were added. In addition, the dummy variable $(D)$ introduced previously, which was used to separate the first and second survey data, is involved in the models.

$\left(D_{1}, D_{2}\right)= \begin{cases}(1,0) & \text { Intersection } 1 \text { is selected } \\ (0,1) & \text { Intersection } 2 \text { is selected } \\ (0,0) & \text { Intersection } 3 \text { is selected }\end{cases}$

For a better fit, only nonlinear models were considered.

Model 3:

rating $=\beta_{03}+\beta_{\mathrm{S} 3} \mathbf{S}+\beta_{13} T_{A}+\beta_{23} T_{A}^{2}+\beta_{33} D_{1}+\beta_{43} D_{2}+\beta_{53} D$ 
TABLE 5 Model Summaries for Individual Intersection Rating

\begin{tabular}{|c|c|c|c|c|}
\hline \multirow[b]{2}{*}{ Variable } & \multicolumn{2}{|l|}{ Model 3} & \multicolumn{2}{|l|}{ Model 4} \\
\hline & Coefficient & $t$-Statistic & Coefficient & $t$-Statistic \\
\hline Constant & 9.58 & 22.2 & 8.33 & 20.4 \\
\hline Gender & -0.028 & -0.154 & -0.086 & -0.468 \\
\hline Age & 0.000 & 0.036 & 0.011 & 1.17 \\
\hline Education & -0.345 & -1.88 & -0.620 & -3.39 \\
\hline Driving & 1.07 & 4.21 & 1.01 & 3.98 \\
\hline Enjoy & -1.21 & -3.92 & -0.917 & -2.98 \\
\hline$T_{A}$ & -0.110 & -15.7 & & \\
\hline$T_{A}^{2}$ & 0.000 & 8.25 & & \\
\hline$T_{P}$ & & & -0.093 & -19.4 \\
\hline$T_{P}^{2}$ & & & 0.000 & 12.7 \\
\hline Indicator for intersection (D1) & 0.125 & 0.527 & -0.021 & -0.095 \\
\hline Indicator for intersection (D2) & -0.222 & -1.16 & -0.157 & -0.820 \\
\hline Indicator for survey (D) & -0.753 & -2.60 & -0.365 & 1.27 \\
\hline
\end{tabular}

NOTE: Bold indicates statistical significance at 95\% confidence interval; significance (two-tailed) for Model 3 is .000 and Model 4 is .000; adjusted $R^{2}$ for Model 3 is . .528 and for Model 4 is .532 .

Model 4:

rating $=\beta_{04}+\beta_{\mathbf{S} 4} \mathbf{S}+\beta_{14} T_{P}+\beta_{24} T_{P}^{2}+\beta_{34} D_{1}+\beta_{44} D_{2}+\beta_{54} D$

The model regression results are presented in Table 5. In a simple comparison of the $R^{2}$ values, the results show that Model 4 fits better than Model 3, indicating that perceived time affects user quality ratings more than actual time. The statistical results also show that education level, driving experience, and driving attitude are significant factors (education level is not significant in Model 3). The results show that there is no difference between three intersections as the $t$-values are $<2$, and there is no significant difference between the first and the second survey data sets.

\section{Arterial Level}

Participants were also required to rate the whole arterial design. Two nonlinear regression models are proposed here to verify whether $T_{A}$ or $T_{P}$ better estimates arterial rating. Similarly, the dummy variable was considered in the models to separate the first and second survey data. The sociodemographic and personality variables are considered in the models

Model 5:

$$
\begin{aligned}
\text { rating }= & \beta_{05}+\beta_{\mathrm{s} 5} \mathbf{S}+\beta_{15} T_{A 1}+\beta_{25} T_{A 1}^{2}+\beta_{35} T_{A 2}+\beta_{45} T_{A 2}^{2}+\beta_{55} T_{A 3} \\
& +\beta_{65} T_{A 3}^{2}+\beta_{75} D
\end{aligned}
$$

Model 6:

$$
\begin{aligned}
\text { rating }= & \beta_{06}+\beta_{\mathrm{S} 6} \mathbf{S}+\beta_{16} T_{P 1}+\beta_{26} T_{P 1}^{2}+\beta_{36} T_{P 2}+\beta_{46} T_{P 2}^{2}+\beta_{56} T_{P 3} \\
& +\beta_{66} T_{P 3}^{2}+\beta_{76} D
\end{aligned}
$$

where $T_{A i}$ and $T_{P i}$ are actual and perceived wait times for intersection $i$.

The model regression results are presented in Table 6 . The results show that Model 6 is more significant than Model 5. When a combi- nation of $T_{A}$ (Model 5) is used to fit the regression model, the correlation between variables in the experimental design requires dropping some of the independent variables from the equation. By comparing the $R^{2}$ values, the model using $T_{P}$ (Model 6) fits better than

\begin{tabular}{|c|c|c|c|c|}
\hline \multirow[b]{2}{*}{ Variable } & \multicolumn{2}{|l|}{ Model 5} & \multicolumn{2}{|l|}{ Model 6} \\
\hline & Coefficient & $t$-Statistic & Coefficient & $t$-Statistic \\
\hline Constant & 6.74 & 7.63 & 6.33 & 8.53 \\
\hline Gender & -0.189 & -0.637 & -0.230 & -0.776 \\
\hline Age & -0.003 & -0.201 & 0.000 & 0.031 \\
\hline Education & -0.107 & -0.361 & -0.283 & 0.950 \\
\hline Driving & 0.794 & 1.87 & 0.770 & 1.85 \\
\hline Enjoy & -1.89 & -3.73 & -1.99 & -3.90 \\
\hline$T_{A}$ & - & - & & \\
\hline$T_{A}^{2}$ & 0.000 & -2.30 & & \\
\hline$T_{A}$ & - & - & & \\
\hline$T_{A}^{2}$ & - & - & & \\
\hline$T_{A}$ & - & - & & \\
\hline$T_{A}^{2}$ & 0.000 & -2.41 & & \\
\hline$T_{P}$ & & & -0.020 & -2.52 \\
\hline$T_{P}^{2}$ & & & 0.000 & 1.65 \\
\hline$T_{P}$ & & & 0.008 & 0.339 \\
\hline$T_{P}^{2}$ & & & 0.000 & -0.844 \\
\hline$T_{P}$ & & & 0.001 & 0.032 \\
\hline$T_{P}^{2}$ & & & 0.000 & -0.826 \\
\hline $\begin{array}{l}\text { Indicator for } \\
\text { survey (D) }\end{array}$ & 0.244 & 0.525 & 0.507 & 1.10 \\
\hline
\end{tabular}
using $T_{A}$ (Model 5). In addition, most of the sociodemographic variables are not significant in the regression models except driving atti-

TABLE 6 Model Summaries for Arterial Rating

Note: Bold indicates statistical significance at $95 \%$ confidence interval; — indicates that collinearity is too small (.000); significance (two-tailed) for Model 5 is .001 and for Model 6 is .000; adjusted $R^{2}$ for Model 5 is .079 and for Model 6 is .117. 
tude. Also, the difference between the first and the second survey data is not significant as the $t$-values in these two models are $<2$.

\section{CONCLUSIONS}

Perceived waiting time at signalized intersections differs from actual waiting time and is significantly affected by signal design. The authors hope that, in the future, signal design will consider user perception. This research aims to answer some related questions about $T_{P}$ based on the preliminary results of a virtual experience SP experiment, which directly collected $T_{P}$ and user ratings of the signal design of three intersections and the arterial that contains them. Statistically analyzing the survey data implies that the perception of waiting time is a function of the actual time, and a quadratic model better describes this relationship. This survey also indicates that there exists a trade-off between total waiting time and individual waiting time before each intersection. Drivers prefer not to spend all their time at one intersection, especially if it has a long wait; people like to feel as if they are making progress. Moreover, the survey data show that $T_{P}$, instead of $T_{A}$, better explains user rankings.

These conclusions are based on two survey studies, but the sample size is relatively small. The robustness of these findings needs to be further demonstrated with larger and more general population samples and multiple methodologies. Also, the current survey did not consider the impact of the number of stops. As indicated in the comments of participants, travelers do care about the total number of stops, and this factor should be considered in future studies. In addition, the survey is based on a traffic simulation. How accurately the scenarios posed here represent real conditions remains to be verified.

\section{ACKNOWLEDGMENTS}

The authors thank Saif Jabari of the University of Minnesota for his help collecting the survey data, Feng Xie of the University of Minnesota for valuable suggestions on data analysis, Yu Han from China for help with website design, and Fengqin Lian of the University of Minnesota for statistics explanation. The authors also appre- ciate the constructive comments offered by the anonymous reviewers of this paper.

\section{REFERENCES}

1. Levinson, D., K. Harder, J. Bloomfield, and K. Carlson. Waiting Tolerance: Ramp Delay vs. Freeway Congestion. Transportation Research F, Vol. 9 , No. 1, 2006, pp. 1-13.

2. Loehlin, J. The Influence of Different Activities on the Apparent Length of Time. Psychological Monographs, Vol. 73, No. 4, 1959, pp. 1-27.

3. Osuna, E. E. The Psychological Cost of Waiting. Journal of Mathematical Psychology, Vol. 29, 1985, pp. 82-105.

4. Cofer, C. N., and M. H. Appley. Motivation: Theory and Research. Wiley, New York, 1964.

5. Becker, G. A Theory of the Allocation of Time. Economic Journal, Vol. 75, 1965, pp. 493-517.

6. Johnson, M. Travel Time and the Price of Leisure. Western Economic Journal, Spring, 1966, pp. 135-145.

7. Oort, C. J. The Evaluation of Traveling Time. Journal of Transport Economics and Policy, Vol. 3, 1969, pp. 279-286.

8. Evans, A. On the Theory of the Valuation and Allocation of Time. Scottish Journal of Political Economy, Vol. 19, 1972, pp. 1-17.

9. DeSerpa, A. A Theory of the Economics of Time. Economic Journal, Vol. 81, 1971, pp. 828-846.

10. Jara-Diaz, S. R., and C. A. Guevara. Behind the Subjective Value of Travel Time Savings: The Perception of Work, Leisure, and Travel from a Joint Mode Choice Activity Model. Journal of Transport Economics and Policy, Vol. 37, No. 1, 2003, pp. 29-46.

11. Mackie, P. J., S. R. Jara-Diaz, and A. S. Fowkes. The Value of Travel Time Savings in Evaluation. Transportation Research E, Vol. 37, No. 2, 2001, pp. 91-106.

12. Bruzelius, N. The Value of Travel Time. Croom Helm, London, 1979.

13. Levinson, D., K. Harder, J. Bloomfield, and K. Winiarczyk. Weighting Waiting: Evaluating Perception of In-Vehicle Travel Time Under Moving and Stopping Conditions. In Transportation Research Record: Journal of the Transportation Research Board, No. 1898, Transportation Research Board of the National Academies, Washington D.C., 2004, pp. 61-68.

14. Zhang, L., F. Xie, and D. Levinson. Variation of Subjective Value of Travel Time on Freeways and Ramp Meters. Presented at 84th Annual Meeting of the Transportation Research Board, Washington, D.C., 2005.

15. Zhang, L., and D. Levinson. Balancing Efficiency and Equity of Ramp Meters. Journal of Transportation Engineering, Vol. 131, No. 6, 2005 , pp. 477-481.

The Traveler Behavior and Values Committee sponsored publication of this paper. 